\title{
Interactive comment on "Mechanistic
} representation of soil nitrogen emissions in the Community Multi-scale Air Quality (CMAQ) model v 5.1” by Quazi Z. Rasool et al.

\section{Anonymous Referee \#2}

Received and published: 10 January 2019

This paper documents a process-based model of soil emissions of $\mathrm{N}$ species (NO, $\mathrm{HONO}, \mathrm{N2O}$, and $\mathrm{NH} 3$ ) implemented in the air-quality model CMAQ. Previous versions of CMAQ used empirical methods to estimate these emissions. The new equations incorporated in CMAQ are based on the biogeochemical ecosystem model DayCent. The new methodology was evaluated for NO2 columns using Ozone Monitoring Instrument satellite retrievals and for other $\mathrm{N}$ species using ground-based monitoring networks. The authors concluded that the new modeling scheme yielded improvements over previous empirical methods, especially in capturing greater spatial variability. The paper addresses modeling questions that fall within the scope of GMD. 
within the scope of EGU. In general, the manuscript describes valid scientific methods to present results (maps, tables, statistical analyses, etc.) and overall suggest a substantial progress in modelling science. While not original, the CMAQ approach uses methods from the DayCent model to simulate soil processes such as nitrification and denitrification known to influence the generation of NOx and other N species. However, not in all cases the results presented are clear and compelling. For example, Figure 3. The authors explain the results due to "likely" causes. Figure $3 \mathrm{c}$ does not convey clearly the results intended by the authors. This part should be clarified. It also appears that the process-based methods introduced in the CMAQ framework cannot be rigorously tested due to lack or old data, which detracts somehow from the considerable efforts made to improve the accuracy in soil $\mathrm{N}$ emission predictions. Presentation quality is fine. Whenever possible, authors should include estimates of estimation or observational errors (e.g. Table 3). Abbreviations used in tables and figures should be explained in the table titles or figure captions. Tables and figures should stand on their own. Since CMAQ already uses EPIC to simulate NH3 bi-directional exchange, the authors should acknowledge recent documentation of process-based denitrification approaches used in EPIC: Izaurralde et al. (2017). Ecol. Modelling 359:349-362 doi: 10.1016/j.ecolmodel.2017.06.007. (see line 481). The methodology and Figure 2 do not describe well the treatment of soil layer processes. EPIC simulates soil $\mathrm{C}$ and $\mathrm{N}$ transformation layer by layer up to 15. Is it the same for DayCent? How are the results from one model past to the other? Are these calculations done for the surface layer? The authors should mention what impact could have an increase in the spatial resolution of the simulation in order to better capture the soil / management heterogeneity.

Interactive comment on Geosci. Model Dev. Discuss., https://doi.org/10.5194/gmd-2018-276, 2018.

Printer-friendly version

Discussion paper 